\title{
Constructing Invariants for Finite Groups
}

\author{
W. Plesken and D. Robertz
}

\section{CONTENTS}

1. Introduction

2. Bigraded Modules

3. The Weyl Algebra

4. Constructing Invariants for $V$ and $V^{*}$ Simultaneously

5. Invariant Vector Fields and Derivations

6. Invariant Poisson Brackets

7. The Group $\mathrm{GL}(2,3)$ with Character $2+\overline{2}$

8. The Group $C_{2} \times \operatorname{PSL}(2,7)$ with Character $3+\overline{3}$ References
2000 AMS Subject Classification: Primary 13A50, 16W22, 17B66, 13N10; Secondary 13P10

Keywords: Invariant theory, computational group theory, invariant differential operators, Janet bases
It is well known that the ring of polynomial invariants of a finite matrix group $G$ becomes more and more messy the further one moves away from groups generated by pseudoreflections, see [Huffman and Sloane 79]. Also, the number of generators has a tendency to become large. In this experimental study, we try to create evidence for the observation that these unpleasant properties might improve if one enlarges the ring of invariants in one way or another. For instance, $G$ might fix a symplectic form. It can be used to turn the ring of invariants into a Lie algebra by introducing a $G$-invariant Poisson bracket. In the absence of an invariant symplectic form, one might still consider the Lie algebra of invariant polynomial vector fields simultaneously with the ring of polynomial invariants. If one is prepared to leave the realm of commutative rings and Lie algebras, one can also take $G$-invariant differential operators into account. Under these additional operations the number of generators necessary to create all invariants is often drastically decreased. A particularly nice situation arises if the group fixes a quadratic form and a symplectic form at the same time, because together they give rise to an endomorphism on the space of homogeneous invariants of any given degree that, for instance, can be used to single out more effective generators in the new sense. In the classical situation of fields of characteristic zero, to which this paper is restricted, the averaging operator is both theoretically and algorithmically an important tool, whose computational feasibility, however, decreases with increasing degrees. The methods presented here might also help to avoid this difficulty.

\section{INTRODUCTION}

This paper starts with an outline of bigraded modules that come up in invariant theory of finite groups. At the end of Section 2, this is applied to reducible groups, in particular, the complexity of constructing the ring of invariants of a reducible finite group is shown to be more or less additive only in the corresponding complexities for the constituent groups. In Section 3, the bigrading approach is applied to the $G$-invariants in the Weyl algebra, and consequences for ordinary invariants, in particular, in the presence of $G$-invariant bilinear forms are investigated. The advantages of constructing the 
invariants of a group and the transposed group at the same time are discussed in Section 4. The observation that invariant polynomial vector fields are very effective in reducing the number of generators for the invariant ring is outlined in Section 5. The Poisson bracket derived from a $G$-invariant symplectic form is discussed in Section 6. Two examples are presented at the end, a small one in Section 7 and one, where standard software (cf. [Bosma et al. 97]) almost reaches its limits, in Section 8.

\section{BIGRADED MODULES}

Throughout this paper $K$ will be the field of complex numbers, $G$ a finite group, $V$ a finitely generated $K G$ module affording the matrix representation $\Delta: G \rightarrow$ $\mathrm{GL}(n, K)$ with respect to some fixed basis, and $V^{*}$ the dual module affording the matrix representation $\Delta^{-t r}$ with respect to the dual basis $x_{1}, \ldots, x_{n}$. Finally, $\operatorname{Irr}(G)$ denotes the set of irreducible characters of $G$.

$\mathbb{Z}$ - or even $\mathbb{Z}_{\geq 0}$-graded modules are quite common in invariant theory of finite groups. For instance, the symmetric algebra $S(V)$ and the polynomial algebra $S\left(V^{*}\right)=K\left[x_{1}, \ldots, x_{n}\right]$ are $\mathbb{Z}_{\geq 0}$-graded $K G$-modules in the obvious way, as well as the Grassmann algebras $\Lambda(V)$ and $\Lambda\left(V^{*}\right)$. For a $\mathbb{Z}$-graded $K G$-module, $M:=\oplus_{i \in \mathbb{Z}} M_{i}$ with finite-dimensional components $M_{i}$, we define the graded character $\chi_{M}:=\sum_{i \in \mathbb{Z}} \chi_{M_{i}} t^{i}$ as a generating function, where $\chi_{M_{i}}$ is the character of $G$ afforded by $M_{i}$. There are two ways to represent these graded characters, namely as class functions of $G$ taking values in generating functions with complex coefficients or as linear combinations of the irreducible characters of $G$ with generating functions which have nonnegative integral coefficients. By extending the usual scalar product of characters, the latter form of graded characters could be written as $\sum_{\chi \in \operatorname{Irr}(G)}\left(\chi_{M}(t), \chi\right) \chi$.

For instance, the graded character of $S(V)$ (respectively $S\left(V^{*}\right)$ ) is given by the expansions of

$$
\begin{aligned}
& \frac{1}{\left(1-\zeta_{1} t\right) \cdots\left(1-\zeta_{n} t\right)}=\frac{1}{\operatorname{det}\left(I_{n}-\Delta(g) t\right)} \\
& \left(\text { respectively } \frac{1}{\left(1-\zeta_{1}^{-1} t\right) \cdots\left(1-\zeta_{n}^{-1} t\right)}\right),
\end{aligned}
$$

where $\zeta_{1}, \ldots, \zeta_{n}$ are the eigenvalues of the action $\Delta(g)$ of $g \in G$ on $V$. Taking the scalar product $\left(\chi_{S\left(V^{*}\right)}, 1_{G}\right)=$ $\left(\chi_{S(V)}, 1_{G}\right)$ of this graded character with the trivial character $1_{G}$ gives the Hilbert series for the (graded) invariant ring $S(V)^{G}$ or $S\left(V^{*}\right)^{G}$ in its well known Molien expression. The reciprocal series of $\chi_{S(V)}$ gives the graded character for $\Lambda(V)$, i.e., $\chi_{S(V)} \cdot \chi_{\Lambda(V)}=1$.

The aim of this section is to extend the above wellknown results to the bigraded case.

Definition 2.1. Let $M$ be a $\mathbb{Z} \times \mathbb{Z}$-graded $K G$-module with finite-dimensional components $M_{(i, j)}$. Then the $b i$ graded character of $M$ is defined by

$$
\chi_{M}\left(t_{1}, t_{2}\right):=\sum_{i, j \in \mathbb{Z}} \chi_{M_{(i, j)}} t_{1}^{i} t_{2}^{j} .
$$

The natural examples we are interested in are of the form $M \otimes N$ where $M=\oplus_{i} M_{i}$ and $N=\oplus_{j} N_{j}$ are $\mathbb{Z}_{\geq 0^{-}}$ graded $K G$-modules with finite-dimensional components. Then $M \otimes N=\oplus_{i, j} M_{i} \otimes N_{j}$ is a bigraded $K G$-module, where all the tensor products are taken over $K$. One obviously has:

Remark 2.2. For $M, N$ as above one has $\chi_{M \otimes N}\left(t_{1}, t_{2}\right)=$ $\chi_{M}\left(t_{1}\right) \chi_{N}\left(t_{2}\right)$.

One such example is $S\left(V^{*}\right) \otimes \Lambda\left(V^{*}\right)$, which, treated in this way, would yield Theorem 5.2.2 of [Benson 93] as a corollary. More relevant for this paper is the following example.

Example 2.3. Let $V=V_{1} \oplus V_{2}$ be a decomposable $K G$ module with direct $K G$-summands $V_{1}, V_{2} \leq V$. Then

$$
S(V)=S\left(V_{1}\right) \otimes S\left(V_{2}\right) \quad \text { and } \quad S\left(V^{*}\right)=S\left(V_{1}^{*}\right) \otimes S\left(V_{2}^{*}\right)
$$

are even bigraded $K G$-algebras. For instance, if $x_{1}, \ldots, x_{k}$ is a basis of $V_{1}^{*}$ and $y_{1}, \ldots, y_{l}$ a basis for $V_{2}^{*}$, then $S\left(V^{*}\right)=K\left[x_{1}, \ldots, x_{k}, y_{1}, \ldots, y_{l}\right]$ and the bidegree is given by the degree in the $x_{i}$ and the degree in the $y_{j}$. By the $G$-elementwise interpretation of Remark 2.2 one gets the bigraded character value for $g \in G$

$$
\begin{aligned}
& \chi_{S(V)}\left(t_{1}, t_{2}\right)(g)= \\
& \frac{1}{\left(1-\zeta_{1} t_{1}\right) \cdots\left(1-\zeta_{k} t_{1}\right)\left(1-\xi_{1} t_{2}\right) \cdots\left(1-\xi_{l} t_{2}\right)}, \\
& \chi_{S\left(V^{*}\right)}\left(t_{1}, t_{2}\right)(g)= \\
& \frac{1}{\left(1-\zeta_{1}^{-1} t_{1}\right) \cdots\left(1-\zeta_{k}^{-1} t_{1}\right)\left(1-\xi_{1}^{-1} t_{2}\right) \cdots\left(1-\xi_{l}^{-1} t_{2}\right)},
\end{aligned}
$$

where $\zeta_{1}, \ldots, \zeta_{k}$ and $\xi_{1}, \ldots, \xi_{l}$ are the eigenvalues of the linear actions $\Delta_{1}(g), \Delta_{2}(g)$ of $g$ on $V_{1}$ and $V_{2}$ respectively. Taking the scalar product with the trivial character $1_{G}$ of 
$G$ gives a generalized Molien formula for the $\mathbb{Z}_{\geq 0}^{2}$-Hilbert series of $S(V)^{G}$ and $S\left(V^{*}\right)^{G}$ :

$$
\begin{aligned}
\left(\chi_{S\left(V_{1} \oplus V_{2}\right)}\left(t_{1}, t_{2}\right), 1_{G}\right) & \\
& :=\sum_{d_{1}, d_{2}} \operatorname{Dim}\left(\left(S\left(V_{1}\right)_{d_{1}} \otimes S\left(V_{2}\right)_{d_{2}}\right)^{G}\right) t_{1}^{d_{1}} t_{2}^{d_{2}} \\
& =\frac{1}{|G|} \sum_{g \in G} \frac{1}{\operatorname{det}\left(I_{k}-\Delta_{1}(g) t_{1}\right) \operatorname{det}\left(I_{l}-\Delta_{2}(g) t_{2}\right)},
\end{aligned}
$$

which is equal to $\left(\chi_{S\left(V_{1}^{*} \oplus V_{2}^{*}\right)}\left(t_{1}, t_{2}\right), 1_{G}\right)$.

Using the characterwise interpretation of Remark 2.2 one gets from

$$
\chi_{S\left(V_{i}\right)}\left(t_{i}\right)=\sum_{\chi \in \operatorname{Irr}(G)}\left(\chi_{S\left(V_{i}\right)}\left(t_{i}\right), \chi\right) \chi
$$

to the formula

$$
\begin{aligned}
\left(\chi_{S(V)}\left(t_{1}, t_{2}\right), 1_{G}\right) & = \\
\sum_{\chi \in \operatorname{Irr}(G)}\left(\chi_{S\left(V_{1}\right)}\left(t_{1}\right), \chi\right)\left(\chi_{S\left(V_{2}\right)}\left(t_{2}\right), \bar{\chi}\right) &
\end{aligned}
$$

because in the double sum over $\operatorname{Irr}(G) \times \operatorname{Irr}(G)$ one has the scalar products $\left(\chi \psi, 1_{G}\right)=(\chi, \bar{\psi})=\delta_{\chi, \bar{\psi}}$. For $S\left(V^{*}\right)$, which is more relevant for us, one of course has the corresponding formula. This formula might be better than the original one above from the point of view of available software, see [The GAP Group 00].

We leave it as an exercise to the reader to deal with multigradings in the case where one has more than two direct summands. The results above will be applied in the next section to Weyl algebras and later on to specific examples.

We finish this section by remarking that the computational complexity for handling $S\left(V_{1}^{*} \oplus V_{2}^{*}\right)$ under the bigrading point of view is essentially the sum of the two complexities for handling $S\left(V_{1}^{*}\right)$ and $S\left(V_{2}^{*}\right)$.

Let us recall that involutive bases [Blinkov et al. 01, Blinkov et al. 03, Cid and Plesken 01] provide a powerful tool for commutative algebra. Given the polynomial algebra $K\left[z_{1}, \ldots, z_{n}\right]$ and a finite set $L \subseteq K\left[z_{1}, \ldots, z_{n}\right]$, the involutive basis algorithm transforms $L$ into another finite set $J \subseteq K\left[z_{1}, \ldots, z_{n}\right]$ which generates the same ideal $\langle L\rangle$ as $L$, but has nice combinatorial properties. For instance, the reduction process of an element of $K\left[z_{1}, \ldots, z_{n}\right]$ modulo $\langle L\rangle$ is unique when using the involutive basis $J$. A characterizing property of $J$ is that by taking $K\left[z_{1}, \ldots, z_{n}\right]$-linear combinations of the elements of $J$ no nonzero element of $\langle L\rangle$ can be produced that is not reducible by elements of $J$ (taking also into account the distinction between multiplicative and nonmultiplicative variables). We mainly use Janet bases [Blinkov et al. 03, Plesken and Robertz 05], which are involutive bases defined by Janet division, but the following techniques are also valid for general involutive bases.

In our setting, with a decomposable $K G$-module $V=$ $V_{1} \oplus V_{2}$ having direct summands $V_{1}, V_{2} \leq V$ as in Example 2.3 , we identify $S\left(V_{1}^{*}\right)$ with $K\left[x_{1}, \ldots, x_{k}\right]$ and $S\left(V_{2}^{*}\right)$ with $K\left[y_{1}, \ldots, y_{l}\right]$. Proposition 2.4 will be applied to the construction of secondary invariants.

Proposition 2.4. Identifying $S\left(V_{1}^{*}\right)=K\left[x_{1}, \ldots, x_{k}\right]$ and $S\left(V_{2}^{*}\right)=K\left[y_{1}, \ldots, y_{l}\right]$ with their embeddings in $S\left(V_{1}^{*} \oplus\right.$ $\left.V_{2}^{*}\right)=K\left[x_{1}, \ldots, x_{k}, y_{1}, \ldots, y_{l}\right]$, we have:

(1) If $p_{1}, \ldots, p_{k}$ are primary invariants for $S\left(V_{1}^{*}\right)^{G}$ and $q_{1}, \ldots, q_{l}$ are primary invariants for $S\left(V_{2}^{*}\right)^{G}$, then $p_{1}, \ldots, p_{k}, q_{1}, \ldots, q_{l}$ form primary invariants for $S\left(V_{1}^{*} \oplus V_{2}^{*}\right)^{G}$.

(2) Let $J_{1}$, respectively $J_{2}$, be a Janet basis for $\left\langle p_{1}, \ldots, p_{k}\right\rangle$ in $S\left(V_{1}^{*}\right)$, respectively for $\left\langle q_{1}, \ldots, q_{l}\right\rangle$ in $S\left(V_{2}^{*}\right)$. A unique coset representative for any element of $S\left(V_{1}^{*} \oplus V_{2}^{*}\right)$ can be obtained by first performing involutive reduction modulo $J_{1}$ in $K\left(y_{1}, \ldots, y_{l}\right)\left[x_{1}, \ldots, x_{k}\right]$ and then for the result modulo $J_{2}$ in $K\left(x_{1}, \ldots, x_{k}\right)\left[y_{1}, \ldots, y_{l}\right]$.

Proof: (1) This is clear. (2) The coset representative is unique if and only if the zero polynomial is the unique representative of the coset given by the ideal $I$ in $S\left(V_{1}^{*} \oplus V_{2}^{*}\right)$ which is generated by the union of $J_{1}$ and $J_{2}$. The latter is satisfied if and only if every element of $I$ is mapped to zero by the successive reductions given in the assertion. If the $y_{j}$, respectively the $x_{i}$, lie in the coefficient field for the reduction modulo $J_{1}$, respectively $J_{2}$, the previous condition is met if and only if the $S$-polynomials of all pairs $(p, q), p \in J_{1}, q \in J_{2}$, involutively reduce to zero (see [Gerdt and Blinkov 98]). These reductions, however, are done as follows, where lt denotes the leading term:

$$
\begin{aligned}
& S(p, q):=\operatorname{lt}(q) p-\operatorname{lt}(p) q \\
& \stackrel{\text { red. } \bmod J_{1}}{\longrightarrow} S(p, q)+(q-\operatorname{lt}(q)) p \\
& \stackrel{\text { red. } \bmod J_{2}}{\longrightarrow} S(p, q)+(q-\operatorname{lt}(q)) p-(p-\operatorname{lt}(p)) q=0 .
\end{aligned}
$$


The proof also reveals that involutive bases for $\left\langle p_{1}, \ldots, p_{k}\right\rangle$ in $S\left(V_{1}^{*}\right)$ and $\left\langle q_{1}, \ldots, q_{l}\right\rangle$ in $S\left(V_{2}^{*}\right)$ can be joined and simply completed (in the sense of Algorithm 15 in [Plesken and Robertz 05]) to yield an involutive basis for the ideal $\left\langle p_{1}, \ldots, p_{k}, q_{1}, \ldots, q_{l}\right\rangle$ in $S\left(V_{1}^{*}\right) \otimes$ $S\left(V_{2}^{*}\right)$, provided the $x_{i}$ are ranked higher than the $y_{j}$. In fact, Proposition 2.4 remains valid outside the context of invariants - it simplifies the computation of involutive bases of tensor products considerably and even makes it superfluous, since involutive reduction can be done successively modulo $J_{1}$ and modulo $J_{2}$.

The application of Proposition 2.4 to invariant theory follows. $\Lambda:=S\left(V_{1}^{*} \oplus V_{2}^{*}\right) /\left\langle p_{1}, \ldots, p_{k}, q_{1}, \ldots, q_{l}\right\rangle$ is a finite-dimensional bigraded commutative $K$-algebra with $G$-action. Representatives $s_{i}$ of a $K$-basis of the algebra $\Lambda^{G}$ of fixed points can be chosen to be $G$-invariant, i.e., to lie in $S\left(V_{1}^{*} \oplus V_{2}^{*}\right)^{G}$, and are known as secondary invariants:

$$
S\left(V_{1}^{*} \oplus V_{2}^{*}\right)^{G}=\bigoplus_{i} K\left[p_{1}, \ldots, p_{k}, q_{1}, \ldots, q_{l}\right] s_{i}
$$

In the present situation, the $s_{i}$ can be chosen to be homogeneous in the bigraded sense. Proposition 2.4 can be used very effectively to decompose any invariant according to $(2-1)$, even if not all of the $s_{i}$ are known already - either one comes up with a decomposition or one has found a new $s_{i}$. The computational trick (see also Algorithm 2 in [Cid and Plesken 01]) is to introduce new parameters $P_{i}$ and $Q_{j}$ as names for the $p_{i}$ and $q_{j}$ for bookkeeping and iterate the simplified Janet reduction of Proposition 2.4 to express any invariant as a polynomial in the $P_{i}$ and $Q_{j}$, where the coefficients are Janet-reduced polynomials in the $x_{i}$ and $y_{j}$. This reduces the decomposition of the new invariant to ordinary Gauss elimination over $K$. As for the dimensions, note that both $S\left(V_{1}^{*}\right) /\left\langle p_{1}, \ldots, p_{k}\right\rangle$ and $S\left(V_{2}^{*}\right) /\left\langle q_{1}, \ldots, q_{l}\right\rangle$ are free $K G$-modules (see [Stanley 79, Proposition 4.9]) if $G$ acts faithfully on both $V_{1}$ and $V_{2}$, in particular, their dimensions are multiples of $|G|$. Therefore, $\Lambda$, as the tensor product over $K$ of the two components, has a rather big dimension, namely the product of the dimensions of the components and $\operatorname{Dim} \Lambda^{G}=\operatorname{Dim} \Lambda /|G|$, which is the number of the secondary invariants $s_{i}$. Examples show that the approach via Algorithm 2 in [Cid and Plesken 01] supersedes the classical methods, even if one ignores the bigrading and gets other primary invariants for $S\left(V_{1}^{*} \oplus V_{2}^{*}\right)^{G}$. The most trivial examples one can look at are given by cyclic groups with one dimensional modules $V_{1}$ and $V_{2}$.

\section{THE WEYL ALGEBRA}

A good way to survey the possibilities of how the various enrichments of the ring of invariants can be described is to start with the Weyl algebra. Since we have already looked at $S\left(V^{*}\right)=K\left[x_{1}, \ldots, x_{n}\right]$ it might be more motivating to define the Weyl algebra as a $K$-algebra of $K$-vector space endomorphisms of $K\left[x_{1}, \ldots, x_{n}\right]$.

The Weyl algebra $W_{n}=W_{n}(K)$ is the noncommutative $K$-algebra of $K$-vector space endomorphisms of $K\left[x_{1}, \ldots, x_{n}\right]$ with generators $x_{1}, \ldots, x_{n}, \partial_{1}, \ldots, \partial_{n}$, where $x_{i}$ stands for the multiplication by $x_{i}$ and $\partial_{i}$ for the partial differentiation with respect to $x_{i}$. The obvious commuting and noncommuting relations

$$
\left[x_{i}, x_{j}\right]=0, \quad\left[\partial_{i}, \partial_{j}\right]=0, \quad\left[\partial_{i}, x_{j}\right]=\delta_{i j}, \quad 1 \leq i, j \leq n,
$$

define a presentation for $W_{n}$ as a $K$-algebra. As a $K$ vector space (not as a $K$-algebra) $W_{n}$ carries a bigrading resulting from the gradings of the two polynomial subalgebras $K\left[x_{1}, \ldots, x_{n}\right]$ and $K\left[\partial_{1}, \ldots, \partial_{n}\right]$, and

$$
W_{n}=K\left[x_{1}, \ldots, x_{n}\right] \otimes K\left[\partial_{1}, \ldots, \partial_{n}\right],
$$

where the $(K-)$ tensor product is realized by the product in $W_{n}$. Note, the original polynomial ring $K\left[x_{1}, \ldots, x_{n}\right]$ is by definition a right $W_{n}$-module. As such, it can also be defined as the residue class module of $W_{n}$ modulo the right ideal generated by $\partial_{1}, \ldots, \partial_{n}$.

Starting again with a $K G$-module $V$ for the finite group $G$ and identifying (the faithful $W_{n^{-}}$ module) $K\left[x_{1}, \ldots, x_{n}\right]$ with $S\left(V^{*}\right)$, the action of $G$ on $K\left[x_{1}, \ldots, x_{n}\right]$ gives rise to an action of $G$ by $K$ algebra automorphisms on $W_{n}$, by conjugation within the ring of vector space endomorphisms of $K\left[x_{1}, \ldots, x_{n}\right]$. One easily checks that the two commutative subalgebras $K\left[x_{1}, \ldots, x_{n}\right]$ and $K\left[\partial_{1}, \ldots, \partial_{n}\right]$ of $W_{n}$ are mapped onto themselves by this action and can be identified with $S\left(V^{*}\right)$ and $S(V)$ respectively as far as the $G$-action is concerned. Note, the $K$-span of $\partial_{1}, \ldots, \partial_{n}$ in $W_{n}$ can be identified with $V$, because it is dual to $V^{*}$, which is the $K$-span of $x_{1}, \ldots, x_{n}$. Also, the bigrading of $W_{n}$ is respected by the action of $G$ so that the results of Section 2 can be used to write down the bigraded character for $W_{n}$.

Now we turn to $G$-fixed points. It should be noted that our primary interest lies in $S\left(V^{*}\right)^{G}$, but $W_{n}^{G}$ will help us get information about $S\left(V^{*}\right)^{G}$. Therefore, the bihomogeneous components $\left(W_{n}^{G}\right)_{\left(d_{1}, d_{2}\right)}$ of $W_{n}$ are of interest to us, since they map $\left(S\left(V^{*}\right)^{G}\right)_{d}$ into $\left(S\left(V^{*}\right)^{G}\right)_{\max (0, d+d 1-d 2)}$. 
Our discussion shows

$$
\begin{aligned}
& \operatorname{Dim}\left(W_{n}^{G}\right)_{\left(d_{1}, d_{2}\right)}= \\
& \text { coefficient of } t_{1}^{d_{1}} t_{2}^{d_{2}} \text { in } M_{V^{*} \oplus V}\left(t_{1}, t_{2}\right),
\end{aligned}
$$

where $M_{V^{*} \oplus V}\left(t_{1}, t_{2}\right):=\left(\chi_{S\left(V^{*} \oplus V\right)}\left(t_{1}, t_{2}\right), 1_{G}\right)$.

Here is an example demonstrating how elements of $W_{n}^{G}$ can help in generating $S\left(V^{*}\right)^{G}$.

Example 3.1. Let the symmetric group $\Sigma_{n}$ act on $V:=$ $K^{n}$ by permuting coordinates in the natural way. It is well known that the induced action on $K\left[x_{1}, \ldots, x_{n}\right]=$ $S\left(V^{*}\right)$ yields an invariant ring generated by $p_{1}:=x_{1}+$ $\ldots+x_{n}, \ldots, p_{n}:=x_{1}^{n}+\ldots+x_{n}^{n}$, in fact, $S\left(V^{*}\right)^{\Sigma_{n}}=$ $K\left[p_{1}, \ldots, p_{n}\right]$ is a polynomial ring in the $p_{i}$, which are algebraically independent. Note, $d:=x_{1}^{2} \partial_{1}+\ldots+x_{n}^{2} \partial_{n} \in$ $\left(W_{n}^{\Sigma_{n}}\right)_{(2,1)}$ is an invariant vector field (to be treated in Section 5), which generates all $p_{i}$ from $p_{1}$ :

$$
d p_{i}=i p_{i+1}, \quad i=1,2, \ldots, n-1 .
$$

Note also, $\delta:=x_{1} \partial_{1}^{2}+\ldots+x_{n} \partial_{n}^{2} \in\left(W_{n}^{\Sigma_{n}}\right)_{(1,2)}$ generates all $p_{i}$ from $p_{n}$ :

$$
\delta p_{i}=i(i-1) p_{i-1}, \quad i=2, \ldots, n .
$$

In very much the same way, as for the pseudoreflection group of degree $n$ isomorphic to the wreath product $C_{k}<\Sigma_{n}$ acting by permuting the standard basis vectors of $V:=\mathbb{C}^{n}$ and multiplying them with $k$ th roots of 1 , the invariant $p_{k}$ together with the invariant vector field $x_{1}^{k+1} \partial_{1}+\ldots+x_{n}^{k+1} \partial_{n}$ can be used to generate the polynomial invariants.

Since the bigraded Hilbert series for the Weyl algebra is symmetric in the two variables, the question arises whether the passage from $\left(W_{n}^{G}\right)_{\left(d_{1}, d_{2}\right)}$ to $\left(W_{n}^{G}\right)_{\left(d_{2}, d_{1}\right)}$ can be made explicit. This is the case and will now be shown separately in the two cases where $V$ and $V^{*}$ are isomorphic, respectively nonisomorphic, as $K G$ modules. The first case has the additional advantage that one gets an explicit epimorphism from $\left(W_{n}^{G}\right)_{\left(d_{1}, d_{2}\right)}$ onto $\left(S\left(V^{*}\right)^{G}\right)_{d_{1}+d_{2}}$ which is very useful for constructing invariants. One word on conventions - we assume that any element of $W_{n}$ or $\left(W_{n}\right)^{G}$ is given as a $K$-linear combination of elements of the form

$$
x^{\alpha} \partial^{\beta}:=x_{1}^{\alpha_{1}} \cdots x_{n}^{\alpha_{n}} \partial_{1}^{\beta_{1}} \cdots \partial_{n}^{\beta_{n}} .
$$

Therefore, by substituting $y_{i}$ for $\partial_{i}$, the elements of $W_{n}$ are in natural one-to-one correspondence with the elements of the polynomial ring $K\left[x_{1}, \ldots, x_{n}, y_{1}, \ldots, y_{n}\right]$.
We abbreviate $\underline{x}$ for both $x_{1}, \ldots, x_{n}$ and the column vector with these entries, analogously for $\underline{\partial}$ and $\underline{y}$.

Proposition 3.2. Let $\Psi: V \times V \rightarrow K$ be a (not necessarily symmetric) nondegenerate $G$-invariant bilinear form on $V$ represented by its Gram matrix $F$, i.e.,

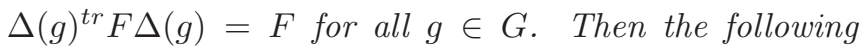
assertions hold:

(1) For each $p\left(x_{1}, \ldots, x_{n}, \partial_{1}, \ldots, \partial_{n}\right) \in\left(W_{n}^{G}\right)_{\left(d_{1}, d_{2}\right)}$ one has $q\left(x_{1}, \ldots, x_{n}, \partial_{1}, \ldots, \partial_{n}\right) \in\left(W_{n}^{G}\right)_{\left(d_{2}, d_{1}\right)}$, where $q(\underline{x}, \underline{y}):=p\left(F^{-1} \underline{y}, F \underline{x}\right)$. In fact, this procedure defines a K-linear involution (i.e., antiautomorphism of order 2) of $W_{n}^{G}$ in the case where $\Psi$ is symmetric and an automorphism of order 2 if $\Psi$ is skewsymmetric.

(2) The map

$$
\begin{aligned}
\left(W_{n}^{G}\right)_{\left(d_{1}, d_{2}\right)} \rightarrow\left(S\left(V^{*}\right)^{G}\right)_{d_{1}+d_{2}}: & p\left(x_{1}, \ldots, x_{n}, \partial_{1}, \ldots, \partial_{n}\right) \\
& \mapsto p(\underline{x}, F \underline{x})
\end{aligned}
$$

is an epimorphism of $K$-vector spaces.

Proof: (1) Proving this is analogous to the slightly more complicated proof of Proposition 3.4. (2) One has $F \Delta(g)=\Delta\left(g^{-1}\right)^{t r} F$ for all $g \in G$. Hence

$p(\Delta(g) \underline{x}, F \Delta(g) \underline{x})=p\left(\Delta(g) \underline{x}, \Delta\left(g^{-1}\right)^{\operatorname{tr}} F \underline{x}\right)=p(\underline{x}, F \underline{x})$

and the map is well defined. To prove surjectivity, let $p(\underline{x}) \in\left(S\left(V^{*}\right)^{G}\right)_{d_{1}+d_{2}}$. Polarizing yields

$$
p(\underline{x}+t \underline{y})=\sum_{i=0}^{d_{1}+d_{2}} p_{i}(\underline{x}, \underline{y}) t^{i},
$$

where $p_{i}(\underline{x}, \underline{y})$ is homogeneous of degree $d_{1}+d_{2}-i$ in the $x_{i}$ and homogeneous of degree $i$ in the $y_{j}$ and $t$ is some independent new variable. Hence, $p_{i}(\underline{x}, \underline{y})$ can be viewed as an element of $S\left(V^{*}\right)_{d_{1}+d_{2}-i} \otimes S\left(V^{*}\right)_{i}$. It is clearly $G$-invariant and by expanding $p((1+t) \underline{x})$ in two ways, one sees that $p_{i}(\underline{x}, \underline{x})=\left(\begin{array}{c}d_{1}+d_{2} \\ i\end{array}\right) p(\underline{x})$. This holds in particular for $i=d_{2}$. Interpret the matrix $F$ as a $K G$ isomorphism from $V$ to $V^{*}$. Now one easily checks that $\left(\begin{array}{c}d_{1}+d_{2} \\ d_{2}\end{array}\right)^{-1} p_{d_{2}}\left(\underline{x}, F^{-1} \underline{\partial}\right)$ is the desired preimage of $p(\underline{x})$ in $\left(W_{n}^{G}\right)_{\left(d_{1}, d_{2}\right)} \equiv\left(S\left(V^{*}\right)_{d_{1}} \otimes S(V)_{d_{2}}\right)^{G}$.

Example 3.3. Let the symmetric group $G:=\Sigma_{n}$ act on $V:=K^{n}$ by coordinate permutations. In Example 3.1, $S\left(V^{*}\right)$ was generated by one vector field $d$ and one invariant $p_{1}$. Using Proposition 3.2 we can now do the same 
with just the vector field $d$ alone (knowing that the standard scalar product is $\Sigma_{n}$-invariant). This is because $d$ produces $\delta$ as well as $p_{3}$ by Proposition 3.2.

Let ${ }^{-}$denote complex conjugation. For a polynomial $p \in K\left[x_{1}, \ldots, x_{n}\right]$ denote by $\bar{p}$ the polynomial obtained from $p$ by applying - to each coefficient. Also for a matrix $F \in K^{n \times n}$, the matrix $\bar{F}$ is obtained from $F$ in the same way.

Proposition 3.4. Let $\Psi: V \times V \rightarrow K$ be a nondegenerate $G$-invariant Hermitian form on $V$ represented by its Gram matrix $F$, i.e., $\overline{\Delta(g)}{ }^{t r} F \Delta(g)=F$ for all $g \in G$. Then for each $p\left(x_{1}, \ldots, x_{n}, \partial_{1}, \ldots, \partial_{n}\right) \in\left(W_{n}^{G}\right)_{\left(d_{1}, d_{2}\right)}$ one has $q\left(x_{1}, \ldots, x_{n}, \partial_{1}, \ldots, \partial_{n}\right) \in\left(W_{n}^{G}\right)_{\left(d_{2}, d_{1}\right)}$, where $q(\underline{x}, \underline{y}):=\bar{p}\left(\bar{F}^{-1} \underline{y}, F \underline{x}\right)$. In fact, this procedure defines a K-semilinear involution of $W_{n}^{G}$.

Proof: One has $F \Delta(g)={\overline{\Delta\left(g^{-1}\right)}}^{t r} F$ for all $g \in G$, which can be manipulated by inverting, applying the field automorphism, and substituting $g^{-1}$ for $g$. Hence

$$
\begin{aligned}
q\left(\Delta(g) \underline{x}, \Delta\left(g^{-1}\right)^{t r} \underline{y}\right) & =\bar{p}\left(\bar{F}^{-1} \Delta\left(g^{-1}\right)^{t r} \underline{y}, F \Delta(g) \underline{x}\right) \\
& =\bar{p}\left(\overline{\Delta(g)} \bar{F}^{-1} \underline{y},{\overline{\Delta\left(g^{-1}\right)}}^{t r} F \underline{x}\right) \\
& =\bar{p}\left(\bar{F}^{-1} \underline{y}, F \underline{x}\right) \\
& =q(\underline{x}, \underline{y})
\end{aligned}
$$

for all $g \in G$, which proves the first half of the assertion. That one has a self-inverting semilinear mapping of $W_{n}$ and also of $W_{n}^{G}$ is obvious to check. That it reverses the order of the multiplication can be seen immediately from the defining relations for $W_{n}$ in the case $F=I_{n}$ and also follows for the general case by checking the defining relations by using a bit more linear algebra.

\section{CONSTRUCTING INVARIANTS FOR $V$ AND $V^{*}$ SIMULTANEOUSLY}

The first possibility to more systematically generate $S\left(V^{*}\right)^{G}$ is E. Fischer's approach from 1911 see [Fischer 11]. He suggested looking at $S\left(V^{*}\right)^{G}$ and $S(V)^{G}$ simultaneously rather than just at $S\left(V^{*}\right)^{G}$. One simply identifies $S\left(V^{*}\right)$ with the $W_{n}$-module $K\left[x_{1}, \ldots, x_{n}\right]$ and $S(V)$ with the subalgebra $K\left[\partial_{1}, \ldots, \partial_{n}\right]$ of $W_{n}$, as we did earlier. Both have the same Hilbert series, $K\left[x_{1}, \ldots, x_{n}\right]^{G}$ becomes a $K\left[\partial_{1}, \ldots, \partial_{n}\right]^{G}$-module, where elements of $\left(K\left[x_{1}, \ldots, x_{n}\right]^{G}\right)_{d}$ are multiplied by elements of $\left(K\left[\partial_{1}, \ldots, \partial_{n}\right]^{G}\right)_{e}$ to get elements of $\left(K\left[x_{1}, \ldots, x_{n}\right]^{G}\right)_{d-e}$. The nice aspect of this approach is that one gets a lot for free, because the invariant differential operators can immediately be constructed from the invariant polynomials (and vice versa). So the next remark is a consequence of Propositions 3.2 and 3.4.

Remark 4.1. Let $\Phi$ be a nondegenerate $G$-invariant bilinear or Hermitian form on $V$ with Gram matrix $F$, i.e., $\Delta(g)^{t r} F \Delta(g)=F$, respectively $\overline{\Delta(g)}^{t r} F \Delta(g)=F$, for all $g \in G$. Then for each invariant $p\left(x_{1}, \ldots, x_{n}\right) \in$ $K\left[x_{1}, \ldots, x_{n}\right]^{G}=S\left(V^{*}\right)^{G}$ one has $p\left(y_{1}, \ldots, y_{n}\right) \in$ $K\left[\partial_{1}, \ldots, \partial_{n}\right]^{G}=S(V)^{G}$ (respectively $\bar{p}\left(y_{1}, \ldots, y_{n}\right) \in$ $K\left[\partial_{1}, \ldots, \partial_{n}\right]^{G}=S(V)^{G}$ ) with $y_{i}:=\sum_{j} f_{i j} \partial_{j}$ (respectively $y_{i}:=\sum_{j} \overline{f_{i j}} \partial_{j}$ ), where the $f_{i j}$ denote the entries of $F^{-1}$.

Fischer himself concentrates on real orthogonal representations, therefore he deals only with the first case with $F=I_{n}$. One must not expect wonders from this approach. For instance, if $p\left(x_{1}, \ldots, x_{n}\right)$ is a quadratic nondegenerate invariant and the matrix $F$ above is just the Hessian matrix of $p$, then $q\left(\partial_{1}, \ldots, \partial_{n}\right)$, obtained from $p$ in the above way, only yields polynomials in $p$ if applied to $p^{k}$ in some power. This is because the full orthogonal group does not have any other invariants.

We shall now demonstrate a more successful example: the group $\mathrm{SL}(2,7)$ of degree 4 . The invariants were worked out by Maschke, see [Maschke 96], and reproduced for the purposes of coding theory in [MacWilliams et al. 72]. We demonstrate how simple it becomes, if one uses Fischer's ideas - all invariants can be constructed from the invariant of degree 4 and the one of degree 6 mainly by using the homogeneous invariant linear differential operator of degree 4 with constant coefficients which can be read off from the invariant of degree 4 .

Example 4.2. The group $G=\mathrm{SL}(2,7)$ has a faithful character of degree 4 . Here is the Molien series:

$$
\frac{1+t^{8}+t^{10}+t^{12}+t^{16}+t^{18}+t^{20}+t^{28}}{\left(1-t^{14}\right)\left(1-t^{8}\right)\left(1-t^{6}\right)\left(1-t^{4}\right)}
$$

The group can be taken to be generated by

$$
\left[\begin{array}{llll}
1 & 0 & 0 & 0 \\
0 & \zeta & 0 & 0 \\
0 & 0 & \zeta^{4} & 0 \\
0 & 0 & 0 & \zeta^{2}
\end{array}\right],\left[\begin{array}{llll}
1 & 0 & 0 & 0 \\
0 & 0 & 0 & 1 \\
0 & 1 & 0 & 0 \\
0 & 0 & 1 & 0
\end{array}\right],
$$




$$
\frac{1}{\sqrt{-7}}\left[\begin{array}{cccc}
1 & 1 & 1 & 1 \\
2 & \zeta+\zeta^{6} & \zeta^{2}+\zeta^{5} & \zeta^{3}+\zeta^{4} \\
2 & \zeta^{2}+\zeta^{5} & \zeta^{3}+\zeta^{4} & \zeta+\zeta^{6} \\
2 & \zeta^{3}+\zeta^{4} & \zeta+\zeta^{6} & \zeta^{2}+\zeta^{5}
\end{array}\right]
$$

where $\zeta$ is a primitive 7 th root of unity. As it stands, the group is not represented by unitary matrices, instead the Gram matrix of the invariant Hermitian form is given by the diagonal matrix $\operatorname{Diag}(2,1,1,1)$. Therefore, any invariant $p\left(x_{1}, x_{2}, x_{3}, x_{4}\right) \in S\left(V^{*}\right)^{G}$ yields the invariant differential operator $\bar{p}\left(\frac{1}{2} \partial_{1}, \partial_{2}, \partial_{3}, \partial_{4}\right)$ according to Remark 4.1. So for instance, the invariant of degree 4

$$
\begin{aligned}
p_{4} & =p_{4}\left(x_{1}, x_{2}, x_{3}, x_{4}\right) \\
& :=2 x_{1}^{4}+6 x_{1} x_{2} x_{3} x_{4}+x_{2}^{3} x_{3}+x_{3}^{3} x_{4}+x_{4}^{3} x_{2}
\end{aligned}
$$

gives rise (up to scaling) to the invariant differential operator of degree 4

$$
P_{4}:=\partial_{1}^{4}+24 \partial_{1} \partial_{2} \partial_{3} \partial_{4}+8 \partial_{2}^{3} \partial_{3}+8 \partial_{3}^{3} \partial_{4}+8 \partial_{4}^{3} \partial_{2} .
$$

One needs to compute one more invariant to get the rest automatically. This is

$$
\begin{aligned}
p_{6}:= & 8 x_{1}^{6}-20 x_{2} x_{3} x_{4} x_{1}^{3}-10\left(x_{2}^{3} x_{3}+x_{3}^{3} x_{4}+x_{4}^{3} x_{2}\right) x_{1}^{2} \\
& -10\left(x_{2}^{2} x_{3}^{3}+x_{3}^{2} x_{4}^{3}+x_{4}^{2} x_{2}^{3}\right) x_{1}-15 x_{2}^{2} x_{3}^{2} x_{4}^{2} \\
& -x_{2} x_{3}^{5}-x_{3} x_{4}^{5}-x_{4} x_{2}^{5} .
\end{aligned}
$$

Since $G$ is perfect, the matrices all have determinant 1 and therefore the Hessian determinant of $p_{4}$ is an invariant, which we call $p_{8}$. Let $p_{14}:=P_{4}\left(p_{6}^{3}\right)$. Using involutive reduction one easily checks that $p_{4}, p_{6}, p_{8}, p_{14}$ form primary invariants. One can also use it to check that the following choices can, for instance, be made for the secondary invariants:

$$
\begin{aligned}
q_{8} & :=P_{4}\left(p_{6}^{2}\right), \\
q_{10} & :=P_{4}\left(p_{14}\right), \\
q_{12} & :=P_{4}\left(p_{8}^{2}\right), \\
q_{16} & :=q_{8}^{2}, \\
q_{18} & :=q_{8} q_{10}, \\
q_{20} & :=q_{10}^{2}, \\
q_{28} & :=q_{10}^{2} q_{8} .
\end{aligned}
$$

\section{INVARIANT VECTOR FIELDS AND DERIVATIONS}

The second approach we want to put forward is the use of invariant vector fields, i.e., working with $\mathcal{V}(V)^{G}$, where

$$
\mathcal{V}(V):=\bigoplus_{i=0}^{\infty}\left(W_{n}\right)_{(i, 1)}
$$

is brought into the game. $\mathcal{V}(V)$ and hence also $\mathcal{V}(V)^{G}$ is closed under the Lie bracket

$$
[a, b]:=a b-b a=\sum_{i}\left(a\left(b_{i}\right)-b\left(a_{i}\right)\right) \partial_{i},
$$

where $a:=\sum a_{i} \partial_{i}, b:=\sum b_{i} \partial_{i} \in \mathcal{V}(V)$, respectively $\mathcal{V}(V)^{G}$. Hence, for $a \in\left(W_{n}\right)_{(i, 1)}^{G}, b \in\left(W_{n}\right)_{(j, 1)}^{G}$ one has $[a, b] \in\left(W_{n}\right)_{(i+j-1,1)}^{G}$ and for $a \in\left(W_{n}\right)_{(i, 1)}^{G}, p \in$ $\left(S\left(V^{*}\right)^{G}\right)_{l}$ one has ap $\in\left(S\left(V^{*}\right)^{G}\right)_{i+l-1}$. This enforces the grading

$$
\mathcal{V}(V)_{i}:=\left(W_{n}\right)_{(i+1,1)}
$$

on $\mathcal{V}(V)$ and hence also on $\mathcal{V}(V)^{G}$. Summarizing we have Remark 5.1.

Remark 5.1. $\mathcal{V}(V)^{G}$ is a $\mathbb{Z}$-graded Lie algebra, i.e., $\left[\left(\mathcal{V}(V)^{G}\right)_{i},\left(\mathcal{V}(V)^{G}\right)_{j}\right] \subseteq\left(\mathcal{V}(V)^{G}\right)_{i+j}$, and $S\left(V^{*}\right)^{G}$ is a graded $\mathcal{V}(V)^{G}$-module. $\left(\mathcal{V}(V)^{G}\right)_{j}=\{0\}$ for $j<-1$ and also for $j=-1$, unless the trivial character occurs in $V$.

To get the Hilbert series of $\mathcal{V}(V)^{G}$ one need not go via the bigraded Hilbert series for $W_{n}^{G}$, but can go directly. $\mathcal{V}(V)$ is isomorphic as a graded $G$-module to $S\left(V^{*}\right) \otimes V$ shifted by -1 and therefore its Hilbert series is given as follows.

Remark 5.2. The Hilbert series of $\mathcal{V}(V)^{G}$ is given by the shifted Molien series

$$
t^{-1}\left(\chi_{S\left(V^{*}\right)}(t), \bar{\chi}\right)=\frac{1}{t|G|} \sum_{g \in G} \frac{\overline{\chi(g)}}{\operatorname{det}\left(I_{n}-\Delta(g) t\right)},
$$

where $\Delta$ is the matrix representation on $V$ with character $\chi$.

Clearly, $\mathcal{V}(V)^{G}$ is also a module for $S\left(V^{*}\right)^{G}$, since an invariant vector field multiplied by an invariant is again an invariant vector field. On the other hand, each vector field acts as a derivation on $S\left(V^{*}\right)^{G}$. Hence, the question arises whether the module $\operatorname{Hom}_{S\left(V^{*}\right)^{G}}\left(\Omega\left(S\left(V^{*}\right)^{G}\right), S\left(V^{*}\right)^{G}\right)$ of all $K$-derivations of the invariant ring coincides with $\mathcal{V}(V)^{G}$, where $\Omega\left(S\left(V^{*}\right)^{G}\right)$ denotes the module of Kähler differentials of the invariant ring $S\left(V^{*}\right)^{G}$. This is a theoretical side issue in view of our aims, since the module of all derivations can only be constructed when one has a presentation of the ring, i.e., at the stage when our job is already done, whereas invariant vector fields can be constructed similarly to invariants. Nevertheless, it is interesting to see from examples that there might be nonpolynomial vector fields as derivations for invariant rings, at least in the presence of pseudoreflections in the group. 
Example 5.3. Let $G$ act faithfully as a pseudoreflection group on $V$. Then $S\left(V^{*}\right)^{G}$ is a polynomial ring $K\left[p_{1}, \ldots, p_{n}\right]$ in $n:=\operatorname{Dim}_{K} V$ independent homogeneous invariants $p_{i}$. Hence, a derivation $\delta$ of $S\left(V^{*}\right)^{G}$ can be obtained by assigning arbitrary values in $S\left(V^{*}\right)^{G}$ to each $p_{i}$. Hence the $\delta_{i}$ defined by $\delta_{j}\left(p_{i}\right):=\delta_{i, j}$ form an $S\left(V^{*}\right)^{G_{-}}$ basis of the module of all derivations. Easy degree considerations show that these are not representable as polynomial vector fields. However, they seem to be representable as vector fields with rational functions as coefficients. For instance, in the case of the symmetric group acting by coordinate permutations and $p_{i}:=x_{1}^{i}+\ldots+x_{n}^{i}$ one gets the Vandermonde determinant in the denominator.

Some work with groups without pseudoreflections shows that $\mathcal{V}(V)^{G}$ may very well coincide with the module of all derivations.

Coming back to the issue of constructing invariants, we shall discuss the effect of $\left(\mathcal{V}(V)^{G}\right)_{0}$. Note first that $V^{*} \otimes V \subset S\left(V^{*}\right) \otimes V \cong \mathcal{V}(V)$ can be identified with $\operatorname{End}_{K}(V)$ via

$$
x_{i} \partial_{j} \mapsto e_{j i},
$$

where $e_{j i}\left(\partial_{k}\right)=\delta_{i k} \partial_{j}$ and $V$ is identified with the vector subspace of $\mathcal{V}(V)$ with basis $\partial_{1}, \ldots, \partial_{n}$. Note that $e_{k l} e_{r s}=\delta_{l r} e_{k s}$. Hence, the Lie bracket induced by the associative product of $\operatorname{End}_{K}(V)$ yields

$$
\left[e_{i j}, e_{k l}\right]=e_{i j} e_{k l}-e_{k l} e_{i j}=\delta_{j k} e_{i l}-\delta_{i l} e_{k j} .
$$

The Lie bracket of the corresponding vector fields is

$$
\begin{aligned}
{\left[x_{j} \partial_{i}, x_{l} \partial_{k}\right]=} & \left(x_{j} \partial_{i}\left(x_{l} \delta_{k i}\right)-x_{l} \partial_{k}\left(x_{j}\right)\right) \partial_{i} \\
& +\left(1-\delta_{i k}\right)\left(x_{j} \partial_{i}\left(x_{l}\right)-x_{l} \partial_{k}\left(x_{j} \delta_{k i}\right)\right) \partial_{k} \\
= & -\delta_{j k} x_{l} \partial_{i}+\delta_{i l} x_{j} \partial_{k}
\end{aligned}
$$

i.e., we have an anti-isomorphism of Lie algebras. Since the brackets are compatible with the group action, passage to the $G$-fixed points yields:

Remark 5.4. $\left(\mathcal{V}(V)^{G}\right)_{0}$ is anti-isomorphic as a Lie algebra to $\left.\operatorname{End}_{K G}(V),[],\right)$.

Example 5.5. Let $G:=C_{r}=\langle g\rangle$ be the cyclic group of order $r$ acting on $V:=K^{n}$ by multiplication with $r$ th roots of unity, i.e., $g \mapsto \zeta_{r} \operatorname{Id}_{V}$, where $\zeta_{r}$ is a primitive $r$ th root of unity. Then the invariants are given by all homogeneous polynomials of degrees divisible by $r$ and a set of generators will be quite big (at least $\operatorname{Dim} S\left(V^{*}\right)_{r}=$ $\left(\begin{array}{c}n+r-1 \\ r\end{array}\right)$ elements). However, $S\left(V^{*}\right)_{d r}$ is a simple module for the Lie algebra $\operatorname{End}_{K G}(V)=\operatorname{End}_{K}(V)=\mathfrak{g l}(n, K)$.
Therefore $x_{1}^{r}$ alone generates $S\left(V^{*}\right)^{G}$ as a $K$-algebra under this Lie algebra action. But, as a Lie algebra, $\mathfrak{g l}(n, K)$ can clearly be generated by two elements. Hence, $S\left(V^{*}\right)^{G}$ can be generated by $x_{1}^{r}$ under the action of two invariant vector fields. In this sense, one has three instead of $\left(\begin{array}{c}n+r-1 \\ r\end{array}\right)$ generators.

One element which is always present in $\left(\mathcal{V}(V)^{G}\right)_{0}$ is $\sum_{i} x_{i} \partial_{i}$ corresponding to the identity element of $\operatorname{End}_{K G}(V)$. It certainly is of no value for generating new invariants, as Euler's identity for applying this element to homogeneous polynomials shows and also since $\left[\sum_{i} x_{i} \partial_{i}, v\right]=l v$ for all $v \in \mathcal{V}(V)_{l}$. In the case where $V$ is absolutely simple as a $K G$-module, the above element is up to $K$-multiples the only one of $\left(\mathcal{V}(V)^{G}\right)_{0}$. Any additional element of $\left(\mathcal{V}(V)^{G}\right)_{0}$ does not only help generating $S\left(V^{*}\right)^{G}$ but also gives more structure to $S\left(V^{*}\right)^{G}$, because one might ask for eigenspaces in each $\left(S\left(V^{*}\right)^{G}\right)_{k}$ or, more generally, decompose it as a $\left(\mathcal{V}(V)^{G}\right)_{0}$-module.

There is one important situation, where one has a closer connection between $S\left(V^{*}\right)^{G}$ and $\mathcal{V}(V)^{G}$ than in the general situation.

Proposition 5.6. Let $V$ be a $K G$-module with nondegenerate $G$-invariant quadratic form $q$. Then the gradient with respect to $q$ defined by

$$
\nabla=\nabla_{q}: S\left(V^{*}\right)^{G} \rightarrow \mathcal{V}(V)^{G}: p \mapsto \sum_{i, j} \hat{q}_{i j} \partial_{i}(p) \partial_{j}
$$

is a derivation of the ring $S\left(V^{*}\right)^{G}$ taking values in $\mathcal{V}(V)^{G}$, where $\left(\hat{q}_{i j}\right)$ is the inverse matrix of the scaled Hessian $\left(\frac{1}{2} \partial_{i} \partial_{j} q\right) \in K^{n \times n}$. It is of degree -2 , i.e., it maps $\left(S\left(V^{*}\right)^{G}\right)_{l}$ to $\left(\mathcal{V}(V)^{G}\right)_{l-2}$. The map

$$
\epsilon=\epsilon_{q}: \mathcal{V}(V)^{G} \rightarrow S\left(V^{*}\right)^{G}: d \mapsto d(q)
$$

is of degree 2 and satisfies $\epsilon(\nabla(p))=k p$ for any $p \in$ $\left(S\left(V^{*}\right)^{G}\right)_{k}$. In particular, $\epsilon$ is surjective from $\left(\mathcal{V}(V)^{G}\right)_{l}$ onto $\left(S\left(V^{*}\right)^{G}\right)_{l+2}$.

Proof: The Hessian is the matrix of a $K G$-isomorphism $V \rightarrow V^{*}$. Hence, the gradient is easily checked to be a $K G$-map of graded $K G$-modules from $S\left(V^{*}\right)$ to $\mathcal{V}(V) \cong$ $S\left(V^{*}\right) \otimes V$ of degree -2 . The derivation property is well known. Hence, one only needs to pass to $G$-fixed points. The last assertion follows from the identity

$$
\left(\nabla p_{1}\right)\left(p_{2}\right)=\left(\nabla p_{2}\right)\left(p_{1}\right) \quad\left(=\sum_{i, j} \partial_{i} p_{1} \partial_{i j} q \partial_{j} p_{2}\right)
$$

applied to $p_{1}:=p, p_{2}:=q$ and $\nabla q=\sum_{i} x_{i} \partial_{i}$. 
Corollary 5.7. Under the assumption of Proposition 5.6 one has a new bilinear (nonassociative) product on $S\left(V^{*}\right)$ defined by

$$
\begin{aligned}
\{,\}_{q}=\{,\} & : S\left(V^{*}\right)^{G} \times S\left(V^{*}\right)^{G} \rightarrow S\left(V^{*}\right)^{G} \\
& :\left(p_{1}, p_{2}\right) \mapsto\left[\nabla p_{1}, \nabla p_{2}\right](q)
\end{aligned}
$$

such that $\left\{\left(S\left(V^{*}\right)^{G}\right)_{a},\left(S\left(V^{*}\right)^{G}\right)_{b}\right\}_{q} \subseteq\left(S\left(V^{*}\right)^{G}\right)_{a+b-2}$ for all $a, b \geq 0$.

Example 5.8. The symmetric group $\Sigma_{5}$ has a threefold transitive permutation representation on six letters. The resulting permutation group can be chosen to be

$$
\langle(1,3,4,2),(3,5)(4,6)\rangle \text {. }
$$

The Molien series for $S\left(V^{*}\right)^{\Sigma_{5}}$, where $V=\mathbb{C}^{6}$ is the permutation module, is given by

$$
\frac{1+t^{6}+t^{8}+t^{9}+t^{10}+t^{12}}{\left(1-t^{6}\right)\left(1-t^{5}\right)\left(1-t^{4}\right)\left(1-t^{3}\right)\left(1-t^{2}\right)(1-t)} \text {. }
$$

The primary invariants can be chosen to be the primary invariants of $S\left(V^{*}\right)^{\Sigma_{6}}$, i.e., $p_{1}:=\sum_{i=1}^{6} x_{i}, \ldots, p_{6}:=$ $\sum_{i=1}^{6} x_{i}^{6}$. One can take gradients with respect to $p_{2}$. In doing this, all the additional information already lies in the secondary invariant $q_{6}:=\sum_{g \in \Sigma_{5}} g\left(x_{1} x_{2} x_{3}^{2} x_{4}^{2}\right)$, because one easily checks that the other secondary invariants can be chosen as $q_{8}:=\left\{q_{6}, p_{4}\right\}$ for degree $8,\left\{q_{6}, p_{5}\right\}$ or $\left\{q_{8}, p_{3}\right\}$ for degree $9,\left\{q_{8}, p_{4}\right\}$ for degree 10 , and finally $q_{6}^{2}$ or $\left\{q_{6}, q_{8}\right\}$ for degree 12 .

\section{INVARIANT POISSON BRACKETS}

In the previous section it was shown that the existence of a quadratic invariant leads to a product on $S\left(V^{*}\right)^{G}$, yielding homogeneous invariants of degree $a+b-2$ from homogeneous factors of degree $a$ and $b$. A similar construction is possible in the presence of a nondegenerate invariant symplectic bilinear form.

Proposition 6.1. Let $V$ be a $K G$-module with nondegenerate $G$-invariant symplectic form s represented by its Gram matrix $S$, i.e., $\Delta(g)^{t r} S \Delta(g)=S$ for all $g \in G$. Then the gradient with respect to $s$ defined by

$$
\nabla=\nabla_{q}: S\left(V^{*}\right)^{G} \rightarrow \mathcal{V}(V)^{G}: p \mapsto \sum_{i, j} \hat{s}_{i j} \partial_{i}(p) \partial_{j}
$$

is a derivation of the ring $S\left(V^{*}\right)^{G}$ taking values in $\mathcal{V}(V)^{G}$, where $\left(\hat{s}_{i j}\right)$ is the inverse matrix of $S$. Moreover, the Poisson bracket

$$
\begin{gathered}
\{,\}_{s}=\{,\}: S\left(V^{*}\right)^{G} \times S\left(V^{*}\right)^{G} \rightarrow S\left(V^{*}\right)^{G} \\
:\left(p_{1}, p_{2}\right) \mapsto\left(\nabla p_{1}\right)\left(p_{2}\right)
\end{gathered}
$$

defines a Lie algebra structure on $S\left(V^{*}\right)$ with $\left\{\left(S\left(V^{*}\right)^{G}\right)_{a},\left(S\left(V^{*}\right)^{G}\right)_{b}\right\}_{s} \subseteq\left(S\left(V^{*}\right)^{G}\right)_{a+b-2}$ for all $a, b \geq 0$.

The proof is straightforward and analogous to the one of Proposition 5.6.

Example 6.2. The quaternion group $Q_{8}$, acting on $K^{2}$ via the matrices

$$
\left(\begin{array}{cc}
i & 0 \\
0 & -i
\end{array}\right), \quad\left(\begin{array}{cc}
0 & 1 \\
-1 & 0
\end{array}\right)
$$

leaves the standard symplectic form invariant. The two primary invariants $p_{1}:=x^{4}+y^{4}, p_{2}:=x^{2} y^{2}$ yield the only missing secondary invariant $q:=\left\{p_{1}, p_{2}\right\}=6 x y\left(x^{4}-y^{4}\right)$.

The following remark will be used repeatedly to replace several generators of the same degree by a single generator.

Remark 6.3. If in the situation of Proposition 6.1 one has a homogeneous invariant $q \in\left(S\left(V^{*}\right)^{G}\right)_{2}$, then one has linear maps

$$
\pi_{q, d}:\left(S\left(V^{*}\right)^{G}\right)_{d} \rightarrow\left(S\left(V^{*}\right)^{G}\right)_{d}: p \mapsto\{q, p\} .
$$

Very often, for small $d \geq 2,\left(S\left(V^{*}\right)^{G}\right)_{d}$ or at least $\left(S\left(V^{*}\right)^{G}\right)_{d} / q\left(S\left(V^{*}\right)^{G}\right)_{d-2}$ is cyclic as a $K\left[\pi_{q, d}\right]$-module, for example, if the space decomposes into a direct sum of one-dimensional eigenspaces. Whether or not an element in this particular situation is not contained in any eigenspace can be seen from its minimal polynomial for $\pi_{q, d}$.

\section{THE GROUP GL $(2,3)$ WITH CHARACTER $2+\overline{2}$}

The group $G:=\operatorname{GL}(2,3)$ has two faithful characters of degree 2 taking values in $K:=\mathbb{Q}[\sqrt{-2}]$. Their sum is rational and has the following Molien series:

$$
\begin{aligned}
& \frac{1+3 t^{6}+4 t^{8}+8 t^{12}+4 t^{16}+3 t^{18}+t^{24}}{\left(1-t^{12}\right)\left(1-t^{8}\right)\left(1-t^{6}\right)\left(1-t^{2}\right)}= \\
& \quad 1+t^{2}+t^{4}+5 t^{6}+10 t^{8}+10 t^{10}+23 t^{12}+\ldots
\end{aligned}
$$

and hence has every potential to have a messy ring of invariants. One expects four primary invariants of degrees $2,6,8$, and 12 , and 24 secondary invariants with degrees $0,6,6,6,8, \ldots$ (according to the numerator) as generators. If one remembers that the two constituent characters correspond to groups generated by pseudoreflections, with degrees of basic invariants 6 and 8, the 
Molien series gets an even worse representation with denominator $\left(1-t^{8}\right)^{2}\left(1-t^{6}\right)^{2}$ and numerator $1+t^{2}+t^{4}+$ $3 t^{6}+6 t^{8}+6 t^{10}+12 t^{12}+6 t^{14}+6 t^{16}+3 t^{18}+t^{20}+t^{22}+t^{24}$ with 48 secondary invariants to be expected. However, this time the Molien series is a specialization of a twovariable Molien series as introduced in Section 2 with denominator $\left(1-t_{1}^{8}\right)\left(1-t_{1}^{6}\right)\left(1-t_{2}^{8}\right)\left(1-t_{2}^{6}\right)$ and numerator

$$
\begin{aligned}
1 & +t_{1}^{12} t_{2}^{12}+\left(t_{1}^{4}+t_{1}^{8}\right)\left(t_{2}^{4}+t_{2}^{8}\right)+\left(t_{1}+t_{1}^{11}\right)\left(t_{2}+t_{2}^{11}\right) \\
& +\left(t_{1}^{5}+t_{1}^{7}\right)\left(t_{2}^{5}+t_{2}^{7}\right)+\left(t_{1}^{6}+t_{1}^{8}+t_{1}^{10}\right)\left(t_{2}^{6}+t_{2}^{8}+t_{2}^{10}\right) \\
& +\left(t_{1}^{2}+t_{1}^{4}+t_{1}^{6}\right)\left(t_{2}^{2}+t_{2}^{4}+t_{2}^{6}\right) \\
& +\left(t_{1}^{3}+t_{1}^{5}+t_{1}^{7}+t_{1}^{9}\right)\left(t_{2}^{3}+t_{2}^{5}+t_{2}^{7}+t_{2}^{9}\right),
\end{aligned}
$$

where the eight summands correspond to the irreducible characters of $G$ of degrees

$$
1,1,2,2,2,3,3,4
$$

in the sense of Section 2. So not only can we count the secondary invariants according to their bidegrees, but also have a definite idea where they come from. To be more specific, let $x_{1}, x_{2}$ be the variables for the first summand and $y_{1}, y_{2}$ the ones for the second, that are dual to the first. Therefore,

$$
q:=x_{1} y_{1}+x_{2} y_{2}
$$

is the invariant corresponding to the term $t_{1} t_{2}$ in the numerator. For the sake of concreteness, let us assume that we know the basic invariants for the components:

$$
\begin{aligned}
p_{6}\left(x_{1}, x_{2}\right) & :=x_{1} x_{2}\left(x_{1}^{4}-x_{2}^{4}\right), p_{8}\left(x_{1}, x_{2}\right) \\
& :=x_{1}^{8}+14 x_{1}^{4} x_{2}^{4}+x_{2}^{8}, p_{6}\left(y_{1}, y_{2}\right), p_{8}\left(y_{1}, y_{2}\right) .
\end{aligned}
$$

Now it turns out that it is not so difficult to construct the invariants of given bidegree and to check whether they are suitable as secondary invariants. The first is done by applying the invariant differential operator $\partial_{y_{1}} \partial_{x_{1}}+\partial_{y_{2}} \partial_{x_{2}}$ corresponding to $x_{1} y_{1}+x_{2} y_{2}$ (the module is self-dual) to $p_{6}\left(x_{1}, x_{2}\right)^{a} p_{8}\left(x_{1}, x_{2}\right)^{b} p_{6}\left(y_{1}, y_{2}\right)^{c} p_{8}\left(y_{1}, y_{2}\right)^{d}$, say $k$-times, to obtain an invariant of bidegree $(6 a+8 b-k, 6 c+8 d-k)$. Multiplying by $q$ increases the bidegree again, by $(1,1)$. To check whether one has secondary invariants, one has to check whether they yield linearly independent elements (in numbers indicated by the numerator polynomial) in

$$
\begin{aligned}
Q:=K\left[x_{1}, x_{2}, y_{1}, y_{2}\right] / \\
\quad\left\langle p_{6}\left(x_{1}, x_{2}\right), p_{8}\left(x_{1}, x_{2}\right), p_{6}\left(y_{1}, y_{2}\right), p_{8}\left(y_{1}, y_{2}\right)\right\rangle,
\end{aligned}
$$

which can easily be done by involutive reduction (see Section 2).
In the process of this construction, one obviously can not expect to obtain secondary invariants corresponding to the irreducible characters. Here is an example. For bidegree $(12,12)$ one can take $q^{12}$ which does not seem to have any relation to the (nontrivial) character of degree 1. But the product of the Jacobi determinants

$$
\frac{\partial\left(p_{6}\left(x_{1}, x_{2}\right), p_{8}\left(x_{1}, x_{2}\right)\right)}{\partial\left(x_{1}, x_{2}\right)} \cdot \frac{\partial\left(p_{6}\left(y_{1}, y_{2}\right), p_{8}\left(y_{1}, y_{2}\right)\right)}{\partial\left(y_{1}, y_{2}\right)}
$$

defines the same coset up to a factor in $\mathbb{Q}$. Anyhow, the construction of the invariants in the proposed form leaves one with twelve secondary invariants (including 1) that have to be multiplied with powers of $q$ according to the Jordan blocks of the multiplication by this polynomial on $Q$, that are of lengths $13,7,7,5,5,5,1,1,1,1,1,1$ with $(0,0),(4,2),(2,4),(4,4),(6,2),(2,6),(11,1),(1,11)$, $(9,3),(3,9),(7,5),(5,7)$ as bidegrees for the generators according to the numerator polynomial. (Usually, 1 does not count as a generator, but $q$ definitely has to be amongst the generators!)

There is one structure on $R:=K\left[x_{1}, x_{2}, y_{1}, y_{2}\right]^{G}$ that we have not yet utilized - the group allows the standard symplectic form and therefore one has the standard Poisson bracket for the invariants

$$
(a, b) \mapsto\{a, b\}:=\sum_{i}\left(\partial_{x_{i}}(a) \partial_{y_{i}}(b)-\partial_{x_{i}}(b) \partial_{y_{i}}(a)\right) .
$$

Taking the Poisson bracket with $q$ leaves the bidegree invariant. In fact, the homogeneous invariants of bidegree $\left(d_{1}, d_{2}\right)$ form the eigenspace for the eigenvalue $d_{2}-d_{1}$ under $\pi_{q, d_{1}+d_{2}}$, as defined in Remark 6.3. Hence, if we allow the Poisson bracket to be part of our invariant generation process, the number of generating invariants can be drastically decreased. For example, for degree 6 , we had four generating invariants, namely one of each of the bidegrees $(6,0),(4,2),(2,4),(0,6)$, say $q_{(6,0)}=p_{6}\left(x_{1}, x_{2}\right), q_{(4,2)}, q_{(2,4)}, q_{(0,6)}=p_{6}\left(y_{1}, y_{2}\right)$. These four can be replaced by one, namely $u:=r_{1} q_{(6,0)}+$ $r_{2} q_{(4,2)}+r_{3} q_{(2,4)}+r_{4} q_{(0,6)}$ with $r_{i} \in K^{*}$, because all four of them lie in the $K$-span of the $\pi_{q, 6}^{i}(u)$ for $i=0,1, \ldots$. Testing whether some invariant $q_{6}$ has nonzero components in each of these eigenspaces can obviously be accomplished by applying $\pi_{q, 6}$ repeatedly to $q_{6}$. If the resulting minimal polynomial of $q_{6}$ for $\pi_{q, 6}$ is divisible by $(t-6)(t+6)(t-2)(t+2)$, then $q_{6}$ has enough components and can therefore be chosen as generating invariant $u$. (Note, the only missing invariant $q^{3}$ is not among the generating invariants.) Similarly, for degree 8 one can replace the five generators by any linear combination of them with nonzero coefficients, because any such combination has these five generators in its span under $K$ and 
$\pi_{q, 8}$. (Note again, the invariants divisible by $q$ can be ignored.) For degree 12 the analogous argument reduces the number of generators from six to one. One can even do without the generator of degree 12 , because it can be chosen to be the Poisson bracket of the two generators of degree 6 and 8 . To prove this, note first the following immediate consequence of Jacobi's identity and the derivation property of the Poisson bracket for the usual product in $K\left[x_{1}, x_{2}, y_{1}, y_{2}\right]$.

Remark 7.1. If $a, b$ are invariants with $\{q, a\}=\lambda_{a} a$ and $\{q, b\}=\lambda_{b} b$ for some $\lambda_{a}, \lambda_{b} \in K$, then $\{q,\{a, b\}\}=$ $\left(\lambda_{a}+\lambda_{b}\right)\{a, b\}$ and $\{q, a b\}=\left(\lambda_{a}+\lambda_{b}\right) a b$.

One checks that all homogeneous invariants of bidegrees $(11,1),(1,11),(9,3),(3,9),(7,5),(5,7)$ can be obtained from Poisson brackets of bihomogeneous invariants of degree 6 and 8 . This proves that the generating invariant of degree 12 can be removed from our generating set. If we call $V$ the $K G$-module having one of the chosen characters, we can summarize as follows in Lemma 7.2.

Lemma 7.2. The ring $R:=S\left(\left(V^{*} \oplus V\right)^{*}\right)^{G}$ is generated as a $K$-vector space together with the usual multiplication and Poisson multiplication $\{$,$\} by the quadratic invari-$ ant $q$ and two further invariants $q_{6} \in R_{6}$ and $q_{8} \in R_{8}$, if

(1) the minimal polynomial of $q_{6}$ under $\pi_{q, 6}$, see Remark 6.3, is divisible by $(t-6)(t+6)(t-2)(t+2)$, and

(2) the minimal polynomial of $q_{8}$ under $\pi_{q, 8}$ is divisible by $t(t-8)(t+8)(t-4)(t+4)$ and the $t$-component of $q_{8}$ is not a $K$-multiple of $q^{4}$.

Testing these properties for $q_{6}$ and $q_{8}$ comes down to straightforward linear dependence checks, where one has to ensure that the criterion does not require a decomposed form of the module, so that it can be applied abstractly as done in Proposition 7.3. For instance, to analyse the condition for $q_{6}$ from a probabilistic point of view, note that $R_{6}$ decomposes into the direct sum of the one-dimensional eigenspaces $E_{\lambda}:=\left\{p \in R_{6} \mid\{q, p\}=\right.$ $\lambda p\}$ with $\lambda \in\{-6,-2,0,2,6\}$. Condition (1) means that $q_{6}$ must not lie in any of the four codimension-1 subspaces $E_{0} \oplus E_{i} \oplus E_{j} \oplus E_{k}$ with $i, j, k$ nonzero. Similar remarks apply to $q_{8}$. In particular, almost all $q_{6} \in R_{6}$ and $q_{8} \in R_{8}$ satisfy the conditions of Lemma 7.2. This reduces the number of generators from $4+47$ in the classical regime to three, namely $q, q_{6}, q_{8}$, if one allows the use of the Poisson bracket.

We now proceed to the $\mathbb{Q} G$-module $W$ with the same character $2+\overline{2}$. The invariant $\operatorname{ring} S\left(W^{*}\right)^{G}$ no longer has the bigraded structure, since $W$ is simple. But it still has the Poisson bracket and a quadratic invariant $\rho$, that is positive definite this time and of discriminant $2^{2}$. Therefore, it makes sense to ask for the nature of the linear action of $\pi_{\rho, d}$, see Remark 6.3, on the spaces of homogeneous invariants of fixed degree $d$. One gets the following characteristic polynomials, the degrees of which are the coefficients in the Taylor expansion of the Molien series.

\begin{tabular}{r|l} 
degree & characteristic polynomial \\
\hline 2,4 & $\chi_{2}:=t$ \\
6 & $\chi_{6}:=\chi_{2} \cdot\left(t^{2}+2 \cdot 2^{2}\right)\left(t^{2}+2 \cdot 6^{2}\right)$ \\
8,10 & $\chi_{8}:=\chi_{6} \cdot t \cdot\left(t^{2}+2 \cdot 4^{2}\right)\left(t^{2}+2 \cdot 8^{2}\right)$ \\
12 & $\chi_{12}:=\chi_{8}^{2} \cdot t^{-1} \cdot\left(t^{2}+2 \cdot 10^{2}\right)\left(t^{2}+2 \cdot 12^{2}\right)$
\end{tabular}

It is clear that the earlier eigenvalues $\lambda=d_{1}-d_{2}$ coming from the bidegrees are replaced by $\sqrt{-2} \lambda$ here. This yields the final result.

Proposition 7.3. The ring $R:=S\left(W^{*}\right)^{G}$ is generated as a $\mathbb{Q}$-vector space together with the usual multiplication and Poisson multiplication $\{$,$\} by the quadratic invariant$ $\rho$ and two further invariants $q_{6} \in R_{6}$ and $q_{8} \in R_{8}$, if

(1) the minimal polynomial of $q_{6}$ under $\pi_{\rho, 6}$, see Remark 6.3, is divisible by $\left(t+2 \cdot 6^{2}\right)\left(t+2 \cdot 2^{2}\right)$, and

(2) the minimal polynomial of $q_{8}$ under $\pi_{\rho, 8}$ is divisible by $t\left(t+2 \cdot 8^{2}\right)\left(t+2 \cdot 4^{2}\right)$ and the $t$-component of $q_{8}$ is not a $\mathbb{Q}$-multiple of $\rho^{4}$.

\section{THE GROUP $C_{2} \times \operatorname{PSL}(2,7)$ WITH CHARACTER $3+\overline{\mathbf{3}}$}

We choose two algebraically conjugate characters of degree 3 of $G:=C_{2} \times \operatorname{PSL}(2,7)$ (which take values in $K:=\mathbb{Q}[\sqrt{-7}])$. As in the preceding example, we study the sum of the two characters. The Molien series for this sum has denominator $\left(1-z^{14}\right)\left(1-z^{6}\right)^{2}\left(1-z^{4}\right)^{2}\left(1-z^{2}\right)$ and numerator $1+3 z^{6}+4 z^{8}+5 z^{10}+6 z^{12}+5 z^{14}+$ $5 z^{16}+6 z^{18}+5 z^{20}+4 z^{22}+3 z^{24}+z^{30}$ which equals $1+z^{2}+3 z^{4}+8 z^{6}+15 z^{8}+30 z^{10}+57 z^{12}+96 z^{14}+$ 


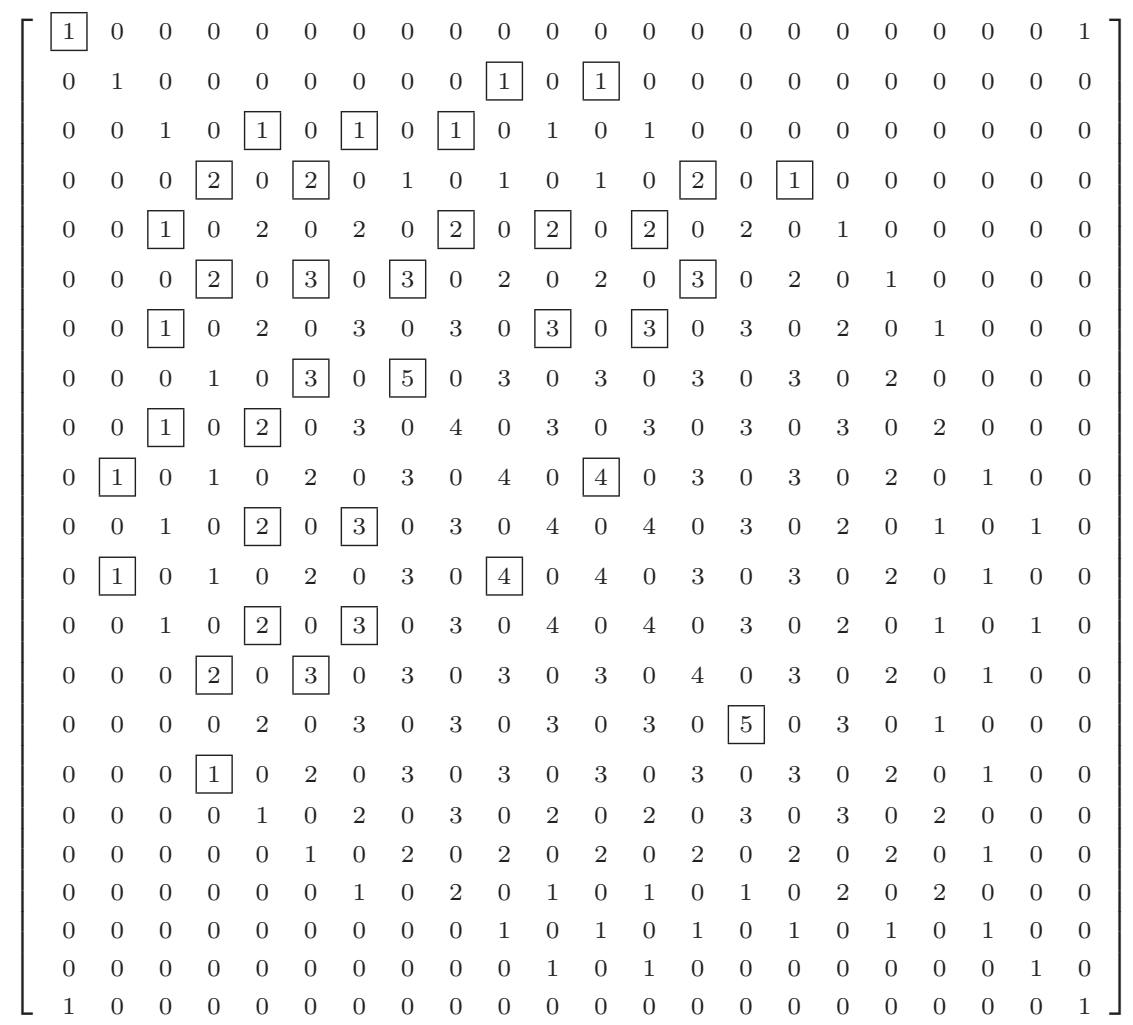

FIGURE 1. Matrix $M, M \in \mathbb{Z}_{\geq 0}^{22 \times 22}$.

$158 z^{16}+252 z^{18}+381 z^{20}+\ldots$. Passing to the bigraded Molien series via the irreducible characters of $G$ yields

$$
\frac{\left(1, t_{1}, t_{1}^{2}, \ldots, t_{1}^{21}\right) M\left(1, t_{2}, t_{2}^{2}, \ldots, t_{2}^{21}\right)^{t r}}{\left(1-t_{1}^{14}\right)\left(1-t_{1}^{6}\right)\left(1-t_{1}^{4}\right)\left(1-t_{2}^{14}\right)\left(1-t_{2}^{6}\right)\left(1-t_{2}^{4}\right)}
$$

with $M \in \mathbb{Z}_{>0}^{22 \times 22}$ given in Figure 1 .

Let $x_{1}, x_{2}, x_{3}$ be the variables for the first summand $V^{*}$ and $y_{1}, y_{2}, y_{3}$ the ones for the second summand $V$. Then we have the quadratic invariant

$$
q:=x_{1} y_{1}+x_{2} y_{2}+x_{3} y_{3},
$$

that corresponds to the second 1 on the diagonal of $M$. For the moment, we assume that we know the primary invariants

$$
\begin{aligned}
& p_{4}\left(x_{1}, x_{2}, x_{3}\right):=x_{1} x_{2}^{3}+x_{2} x_{3}^{3}+x_{3} x_{1}^{3}, \\
& p_{6}\left(x_{1}, x_{2}, x_{3}\right), \quad p_{14}\left(x_{1}, x_{2}, x_{3}\right), \quad p_{4}\left(y_{1}, y_{2}, y_{3}\right), \\
& p_{6}\left(y_{1}, y_{2}, y_{3}\right), \quad p_{14}\left(y_{1}, y_{2}, y_{3}\right),
\end{aligned}
$$

where $p_{6}$ is the Hessian determinant of $p_{4}$, and $p_{14}$ is given by (see [Burnside 55])

$$
p_{14}\left(x_{1}, x_{2}, x_{3}\right):=\left|\begin{array}{cccc}
\frac{\partial^{2} p_{4}}{\partial x_{1}^{2}} & \frac{\partial^{2} p_{4}}{\partial x_{1} \partial x_{2}} & \frac{\partial^{2} p_{4}}{\partial x_{1} \partial x_{3}} & \frac{\partial p_{6}}{\partial x_{1}} \\
\frac{\partial^{2} p_{4}}{\partial x_{1} \partial x_{2}} & \frac{\partial^{2} p_{4}}{\partial x_{2}^{2}} & \frac{\partial^{2} p_{4}}{\partial x_{2} \partial x_{3}} & \frac{\partial p_{6}}{\partial x_{2}} \\
\frac{\partial^{2} p_{4}}{\partial x_{1} \partial x_{3}} & \frac{\partial^{2} p_{4}}{\partial x_{2} \partial x_{3}} & \frac{\partial^{2} p_{4}}{\partial x_{3}} & \frac{\partial p_{6}}{\partial x_{3}} \\
\frac{\partial p_{6}}{\partial x_{1}} & \frac{\partial p_{6}}{\partial x_{2}} & \frac{\partial p_{6}}{\partial x_{3}} & 0
\end{array}\right| .
$$

(However, the latter construction is not needed, see below.) Again, multiplication by $q$ increases the bidegree by $(1,1)$ and the corresponding invariant differential operator

$$
D_{q}^{k}:=\left(\partial_{y_{1}} \partial_{x_{1}}+\partial_{y_{2}} \partial_{x_{2}}+\partial_{y_{3}} \partial_{x_{3}}\right)^{k}
$$

maps $p_{4}\left(x_{1}, x_{2}, x_{3}\right)^{a} \quad p_{6}\left(x_{1}, x_{2}, x_{3}\right)^{b} \quad p_{14}\left(x_{1}, x_{2}, x_{3}\right)^{c}$ $p_{4}\left(y_{1}, y_{2}, y_{3}\right)^{d} p_{6}\left(y_{1}, y_{2}, y_{3}\right)^{e} p_{14}\left(y_{1}, y_{2}, y_{3}\right)^{f}$ to an invariant of bidegree $(4 a+6 b+14 c-k, 4 d+6 e+14 f-k)$. In matrix $M$ the number for secondary invariants of bidegree $(i, j)$ is boxed if and only if there is a secondary invariant of this bidegree that is not obtained as a product of a secondary invariant of lower bidegree with a power of $q$ (up to a constant factor). Only in bidegree $(7,7)$ two new secondary invariants $s_{7,7}, s_{7,7}^{\prime}$ are found, all other boxes demanding only one. The lengths of the Jordan blocks of multiplication by $q$ on $K[\underline{x}, \underline{y}] /\left\langle p_{4}(\underline{x}), p_{6}(\underline{x}), p_{14}(\underline{x}), p_{4}(\underline{y}), p_{6}(\underline{y}), p_{14}(\underline{y})\right\rangle$ can easily be read off from $M$; for instance, the corresponding length for the new invariant $s_{3,3}$ of bidegree $(3,3)$ is 16 .

Since $G$ allows the standard symplectic form, the Poisson bracket

$$
(a, b) \mapsto\{a, b\}:=\sum_{i}\left(\partial_{x_{i}}(a) \partial_{y_{i}}(b)-\partial_{x_{i}}(b) \partial_{y_{i}}(a)\right)
$$


maps pairs of invariants to invariants again. The eigenspaces of $\pi_{q, d}$ are analogous to those in Section 7. For the generation of $S\left(\left(V^{*} \oplus V\right)^{*}\right)^{G}$ it is advantageous to choose invariants $q_{4}$ and $q_{6}$ in degrees 4 and 6 having components in the eigenspaces of eigenvalues $-4,4$ and $0,2,6$ respectively, where the 0-component of $q_{6}$ is not a $K$-multiple of $q^{3}$. Then one can check that all but two of the "boxed" secondary invariants in $M$ as well as $p_{14}\left(x_{1}, x_{2}, x_{3}\right)$ and $p_{14}\left(y_{1}, y_{2}, y_{3}\right)$ can be obtained from Poisson brackets of invariants of appropriate lower bidegrees, the exceptions being generating invariants for bidegrees $(2,6)$ and $(6,2)$. However, using the invariant differential operator $D_{q}$ we succeed by choosing

$$
s_{2,6}:=D_{q}^{2}\left(p_{4}(\underline{x}) p_{4}(\underline{y})^{2}\right), \quad s_{6,2}:=D_{q}^{2}\left(p_{4}(\underline{x})^{2} p_{4}(\underline{y})\right) .
$$

More precisely, we remark that, except for $s_{2,6}, s_{6,2}, s_{7,7}^{\prime}$, $s_{14,14}$, all other generating secondary invariants can be constructed as Poisson brackets $\left\{p_{1}, p_{2}\right\}$, where $p_{1}, p_{2}$ are primary or secondary invariants that are already computed. Only the last two exceptions involve products of invariants inside the Poisson bracket; they may be chosen as follows:

$$
s_{7,7}^{\prime}:=\left\{s_{5,3}, s_{2,4} \cdot q\right\}, \quad s_{14,14}:=\left\{s_{8,2} \cdot s_{3,3}, s_{2,8} \cdot q^{2}\right\} .
$$

We summarize in Lemma 8.1.

Lemma 8.1. The ring $R:=S\left(\left(V^{*} \oplus V\right)^{*}\right)^{G}$ is generated as a $K$-vector space together with the usual multiplication and Poisson multiplication $\{$,$\} by the quadratic invari-$ ant $q$ and two further invariants $q_{4} \in R_{4}$ and $q_{6} \in R_{6}$, if

(1) the minimal polynomial of $q_{4}$ under $\pi_{q, 4}$, see Remark 6.3, is divisible by $(t-4)(t+4)$, and

(2) the minimal polynomial of $q_{6}$ under $\pi_{q, 6}$ is divisible by $t(t-6)(t+6)(t-2)(t+2)$, the $t$-component of $q_{6}$ not being a $K$-multiple of $q^{3}$.

As discussed in Section 7, a random choice of $q_{4}$ and $q_{6}$ most likely gives a generating set in the sense of Lemma 8.1, so that the number of generators for $S\left(\left(V^{*} \oplus V\right)^{*}\right)^{G}$ is decreased by Lemma 8.1 from $4+335$ to three, if one allows the use of $\{$,$\} .$

For the $\mathbb{Q} G$-module $W$ with the same character $3+\overline{3}$, we have a quadratic invariant $\rho$ which gives rise to a map $\pi_{\rho, d}$ on $S\left(W^{*}\right)_{d}^{G}$, see Remark 6.3. The earlier eigenvalues $\lambda=d_{1}-d_{2}$ are replaced by $\sqrt{-7} \lambda$ here. We finish similar to Section 7:
Proposition 8.2. The ring $R:=S\left(W^{*}\right)^{G}$ is generated as a $\mathbb{Q}$-vector space together with the usual multiplication and Poisson multiplication $\{$,$\} by the quadratic invariant$ $\rho$ and two further invariants $q_{4} \in R_{4}$ and $q_{6} \in R_{6}$, if

(1) the minimal polynomial of $q_{4}$ under $\pi_{\rho, 4}$, see Remark 6.3, is divisible by $\left(t+7 \cdot 4^{2}\right)$, and

(2) the minimal polynomial of $q_{6}$ under $\pi_{\rho, 6}$ is divisible by $t\left(t+7 \cdot 6^{2}\right)\left(t+7 \cdot 2^{2}\right)$ and the $t$-component of $q_{6}$ is not a $\mathbb{Q}$-multiple of $\rho^{3}$.

\section{REFERENCES}

[Benson 93] D. J. Benson. Polynomial Invariants of Finite Groups, LMS Lecture Notes, 190. Cambridge, UK: Cambridge Univ. Press, 1993.

[Blinkov et al. 01] Y. A. Blinkov, V. P. Gerdt, and D. A. Yanovich. "Construction of Janet Bases, II. Polynomial Bases." In Proceedings of Computer Algebra in Scientific Computing, CASC 2001, edited by V. G. Ganzha, E. W. Mayr, E. V. Vorozhtsov, pp. 249-263. Berlin-HeidelbergNew York: Springer-Verlag, 2001.

[Blinkov et al. 03] Y. A. Blinkov, C. F. Cid, V. P. Gerdt, W. Plesken, and D. Robertz. "The MAPLE Package 'Janet': I. Polynomial Systems." In Proceedings of Computer Algebra in Scientific Computing CASC 2003, edited by V. G. Ganzha, E. W. Mayr, and E. V. Vorozhtsov, pp. 31-40. Garching, Germany: Institut für Informatik, Technische Universität München, 2003. Also available from World Wide Web (http://wwwb.math. rwth-aachen.de/Janet/).

[Bosma et al. 97] W. Bosma, J. J. Cannon, and C. Playoust. "The Magma Algebra System I: The User Language." J. Symb. Comp. 24 (1997), 235-265. Also available from World Wide Web (http://magma.maths.usyd.edu.au/ magma/MagmaInfo.html).

[Burnside 55] W. Burnside. Theory of Groups of Finite Order, Second edition. New York: Dover Publications, 1955.

[Cid and Plesken 01] C. F. Cid and W. Plesken. "Invariants of Finite Groups and Involutive Division." In Proceedings of Computer Algebra in Scientific Computing, CASC 2001, edited by V. G. Ganzha, E. W. Mayr, E. V. Vorozhtsov, pp. 122-135. Berlin-Heidelberg-New York: Springer-Verlag, 2001.

[Derksen and Kemper 02] H. Derksen and G. Kemper. Computational Invariant Theory, Encyclopedia of Mathematical Sciences, 130. Berlin: Springer, 2002.

[Fischer 11] E. Fischer. "Über algebraische Modulsysteme und lineare homogene Differentialgleichungen mit konstanten Koeffizienten." Journ. reine u. angew. Math. 140 (1911), 48-81. 
[The GAP Group 00] The GAP Group. GAP — Groups, Algorithms, and Programming, Version 4.2. Aachen, St Andrews, 1999. Also available from World Wide Web (http://www.gap-system.org).

[Gerdt and Blinkov 98] V. P. Gerdt and Y. A. Blinkov. "Involutive Bases of Polynomial Ideals." Mathematics and Computers in Simulation 45 (1998), 519-541.

[Huffman and Sloane 79] W. C. Huffman and N. J. A. Sloane. "Most Primitive Groups Have Messy Invariants." Advances in Math. 32 (1979), 118-127.

[MacWilliams et al. 72] F. J. MacWilliams, C. L. Mallows, and N. J. Sloane. "Generalizations of Gleason's Theorem on Weight Enumerators of Self-Dual Codes." IEEE Transact. on Inform. Theory, IT-18:6 (1972), 794-805.
[Mallows and Sloane 73] C. L. Mallows and N. J. A. Sloane. "On the Invariants of a Linear Group of Order 336." Proc. Cambridge Philosophical Soc. 74 (1973), 435-440.

[Maschke 96] H. Maschke. "Invariants of a Group of $2 \cdot 168$ Linear Quaternary Substitutions." In Papers Read at the Int. Math. Congr, Chicago 1893, pp. 175-186. New York: MacMillan, 1896.

[Plesken and Robertz 05] W. Plesken and D. Robertz. "Janet's Approach to Presentations and Resolutions for Polynomials and Linear PDEs." Arch. Math. 84:1 (2005), 22-37.

[Stanley 79] R. P. Stanley. "Invariants of Finite Groups." Bull. AMS 1:3 (1979), 475-511.

W. Plesken, Lehrstuhl B für Mathematik, RWTH Aachen, Templergraben 64, 52062 Aachen, Germany (plesken@momo.math.rwth-aachen.de)

D. Robertz, Lehrstuhl B für Mathematik, RWTH Aachen, Templergraben 64, 52062 Aachen, Germany (daniel@momo.math.rwth-aachen.de)

Submitted May 27, 2004; accepted December 27, 2004. 